PROCEEDINGS OF THE

AMERICAN MATHEMATICAL SOCIETY

Volume 132, Number 3, Pages 847-852

S 0002-9939(03)07157-0

Article electronically published on July 7, 2003

\title{
ON RAKHMANOV'S THEOREM FOR JACOBI MATRICES
}

\author{
SERGEY A. DENISOV
}

(Communicated by Andreas Seeger)

\begin{abstract}
We prove Rakhmanov's theorem for Jacobi matrices without the additional assumption that the number of bound states is finite. This result solves one of Nevai's open problems.
\end{abstract}

Consider a measure $d \mu$ with bounded support in $\mathbb{R}$. Assume that it has infinitely many growth points. Let $\left\{p_{k}\right\}(k=0,1, \ldots)$ be the system of polynomials orthonormal with respect to that measure, i.e.,

$$
\int_{-\infty}^{\infty} p_{k}(x) p_{m}(x) d \mu(x)=\delta_{k, m}, \quad \operatorname{deg} p_{l}(x)=l .
$$

It is known [1] that the following recurrence relations hold:

$$
\begin{aligned}
& b_{-1} p_{0}(x)=1, \quad v_{0} p_{0}(x)+b_{0} p_{1}(x)=x p_{0}(x), \\
& b_{k-1} p_{k-1}(x)+v_{k} p_{k}(x)+b_{k} p_{k+1}(x)=x p_{k}(x), \quad k=1,2, \ldots,
\end{aligned}
$$

with $b_{k}>0, k=-1,0, \ldots$, and $v_{k}$ real. Thus, for any fixed $x, p_{k}$ are generalized eigenvectors of the following Jacobi matrix [1]:

$$
J=\left[\begin{array}{ccccc}
v_{0} & b_{0} & 0 & 0 & \ldots \\
b_{0} & v_{1} & b_{1} & 0 & \ldots \\
0 & b_{1} & v_{2} & b_{2} & \ldots \\
0 & 0 & b_{2} & v_{3} & \ldots \\
\ldots & \ldots & \ldots & \ldots & \ldots
\end{array}\right]
$$

The corresponding operator $J$ is bounded and self-adjoint in the Hilbert space $\ell^{2}\left(\mathbb{Z}^{+}\right)$. And, vice-versa, we can associate to any real Jacobi matrix with positive off-diagonal entries a certain measure on the line (spectral measure). In a general situation, a limit circle case might occur such that this measure is non-unique. But in the limit point case, this measure is uniquely defined. If the corresponding self-adjoint operator $J$ is bounded, then the measure has bounded support. In this paper, we consider only that case. We introduce the following notation: $\sigma(A)$ denotes the spectrum of the self-adjoint bounded operator $A, \sigma_{e s s}(A)$ its essential spectrum, and $\|A\|$ the norm of the operator. The following theorem was proved by Nevai.

Received by the editors October 16, 2002 and, in revised form, November 8, 2002.

2000 Mathematics Subject Classification. Primary 47B36.

(C)2003 American Mathematical Society 
Theorem 1 (Nevai 7], 6]). If $\sigma_{\text {ess }}(J)=[-1,1]$, where $J$ has a finite number of eigenvalues outside the segment $[-1,1]$ and, for the corresponding spectral measure $\mu, \mu^{\prime}(x)>0$ for a.e. $x \in[-1,1]$, then $v_{k} \rightarrow 0, b_{k} \rightarrow 1 / 2$ as $k \rightarrow \infty$.

The following open question was posed in [6] (Conjecture 2.7):

Is the statement of the theorem true without the second assumption on J, i.e., for the case when one has an infinite number of eigenvalues (bound states) outside the segment $[-1,1]$ ?

The main result of our short note is the affirmative answer to this question. To prove that, we will need one auxiliary lemma.

Lemma 1. Consider a bounded self-adjoint operator $A$ in the Hilbert space, such that $\sigma_{\text {ess }}(A)=[a, b]$. Let $\left\{e_{j}\right\}, j=1,2, \ldots$, be the orthonormal basis, and $A_{n}$ the self-adjoint bounded operator, generated by the quadratic form $(A f, f)$, where $f$ is orthogonal to $\left\{e_{1}, \ldots, e_{n}\right\}$. Then $\inf \sigma\left(A_{n}\right) \rightarrow a$, and $\sup \sigma\left(A_{n}\right) \rightarrow b$ as $n \rightarrow \infty$.

Proof. We will only show that $\inf \sigma\left(A_{n}\right) \rightarrow a$ as $n \rightarrow \infty$. The other statement can be proved similarly. Let $\lambda_{j}, \psi_{j}(j=1,2, \ldots)$ denote eigenvalues and the corresponding normed eigenfunctions, such that $\lambda_{1} \leq \lambda_{2} \leq \cdots<a,\left\|\psi_{j}\right\|=1$. Consider any vector $f$ from the Hilbert space. By the spectral theorem, $f=\sum_{j=1}^{\infty}\left(f, \psi_{j}\right) \psi_{j}+f_{c}$ and

$$
\begin{aligned}
(A f, f) \geq a\left(f_{c}, f_{c}\right)+\sum_{j=1}^{\infty} \lambda_{j}\left|\left(f, \psi_{j}\right)\right|^{2} & =a\left[\left(f_{c}, f_{c}\right)+\sum_{j=1}^{\infty}\left|\left(f, \psi_{j}\right)\right|^{2}\right] \\
+ & \sum_{j=1}^{\infty}\left(\lambda_{j}-a\right)\left|\left(f, \psi_{j}\right)\right|^{2}=a\|f\|^{2}+\sum_{j=1}^{\infty}\left(\lambda_{j}-a\right)\left|\left(f, \psi_{j}\right)\right|^{2} .
\end{aligned}
$$

Notice that for fixed $j,\left(f, \psi_{j}\right) \rightarrow 0$ uniformly in $f \in\{\|f\| \leq 1$ and $f$ is orthogonal to $\left.\left\{e_{1}, \ldots, e_{n}\right\}\right\}$ as $n \rightarrow \infty$. Indeed,

$$
\left(f, \psi_{j}\right)=\sum_{k=1}^{\infty}\left(f, e_{k}\right) \overline{\left(\psi_{j}, e_{k}\right)}=\sum_{k=n+1}^{\infty}\left(f, e_{k}\right) \overline{\left(\psi_{j}, e_{k}\right)} .
$$

Consequently, by the Cauchy inequality,

$$
\left|\left(f, \psi_{j}\right)\right| \leq\|f\|\left(\sum_{k=n+1}^{\infty}\left|\left(\psi_{j}, e_{k}\right)\right|^{2}\right)^{1 / 2} \rightarrow 0
$$

as $n \rightarrow \infty$. By the variational principle,

$$
\inf \sigma\left(A_{n}\right)=\inf _{\|f\|=1,\left(f, e_{j}\right)=0, j=1, \ldots, n}(A f, f) .
$$

By the Courant-Hilbert variational principle [11], inf $\sigma\left(A_{n}\right) \leq \lambda_{n+1}$. We know that $\lambda_{j} \rightarrow a$ as $j \rightarrow \infty$. Consequently, from (3), we have $\inf \sigma\left(A_{n}\right) \rightarrow a$ as $n \rightarrow \infty$.

Let $\psi(x \in[-1,1], \psi(-1)=0)$ be a non-decreasing bounded function with an infinite number of growth points. Consider the system of polynomials $p_{k}(k=0,1, \ldots)$ orthonormal with respect to the measure $d \psi$ on the segment $[-1,1]$. Introduce the function

$$
\sigma(\theta)=\left\{\begin{array}{cc}
-\psi(\cos \theta), & 0 \leq \theta \leq \pi \\
\psi(\cos \theta), & \pi \leq \theta \leq 2 \pi
\end{array}\right.
$$


which is bounded and non-decreasing on $[0,2 \pi]$. Consider the polynomials $\phi_{k}(z)=$ $\alpha_{k} z^{k}+\cdots\left(\alpha_{k}>0\right)$ orthonormal on the circle with respect to the measure $d \sigma$, i.e.,

$$
\frac{1}{2 \pi} \int_{0}^{2 \pi} \phi_{k}(z) \overline{\phi_{m}(z)} d \sigma(\theta)=\delta_{k, m}, \quad z=e^{i \theta} .
$$

$\phi_{n}$ are related to $p_{k}$ by the formula

$$
p_{k}(x)=\frac{\phi_{2 k}(z)+\phi_{2 k}^{*}(z)}{\sqrt{2 \pi\left[1+\alpha_{2 k}^{-1} \phi_{2 k}(0)\right]}} z^{-k}, \quad k=0,1, \ldots,
$$

where $x=\left(z+z^{-1}\right) / 2$ and $\phi_{k}^{*}(z)=z^{k} \overline{\phi_{k}\left(\bar{z}^{-1}\right)}$ [12], 3]. Calculations yield the following identities [3], 4]:

$$
\begin{gathered}
b_{k}=\frac{1}{2} \sqrt{\left(1-a_{2 k-1}\right)\left(1-a_{2 k}^{2}\right)\left(1+a_{2 k+1}\right)}, \\
v_{k}=-\frac{1}{2}\left[a_{2 k-2}\left(1+a_{2 k-1}\right)-a_{2 k}\left(1-a_{2 k-1}\right)\right], \quad k \geq 1,
\end{gathered}
$$

where $a_{k}=-\alpha_{k+1}^{-1} \phi_{k+1}(0)$ are the so-called reflection coefficients for $d \sigma$ (parameters of the system [3]). In our particular case, $a_{k}$ are real numbers. Notice that multiplication of the function $\psi$ by the positive constant leads to the multiplication of $p_{n}(x), \sigma(\theta), \phi_{n}(z)$ by certain constants, but it does not change $b_{n}, v_{n}, a_{n}$, i.e., parameters of the orthogonal systems.

The following remarkable theorem was first proved by Rakhmanov [10]. Later, various proofs were given by other authors.

Theorem 2 (Rakhmanov [10], [5]). If $\sigma^{\prime}(\theta)>0$ for a.e. $\theta \in[0,2 \pi]$, then $a_{k} \rightarrow 0$ as $k \rightarrow \infty$.

We will need the following generalization of this result.

Theorem 3. If $\mathbb{K} \equiv\left\{\theta \in[0,2 \pi): \sigma^{\prime}(\theta)>0\right\}$, then

$$
\limsup _{n \rightarrow \infty}\left|a_{n}\right|^{2} \leq 8\left(1-\left(\frac{|\mathbb{K}|}{2 \pi}\right)^{3 / 2}\right),
$$

where $|\mathbb{K}|$ means the Lebesgue measure of a set $\mathbb{K}$.

The proof can be obtained by a slight modification of the arguments given in [5]. We will also need the following well-known result (see, e.g., [5]).

Lemma $2([5])$. If $\nu$ is a measure on $\mathbb{R}$ singular with respect to Lebesgue measure, then there exists a sequence of continuous functions $\left\{h_{n}\right\}$ such that $0<h_{n}(x) \leq 1$ for all $x \in \mathbb{R}$,

$$
\lim _{n \rightarrow \infty} h_{n}(x)=1
$$

for a.e. $x$, and

$$
\lim _{n \rightarrow \infty} \int_{-\infty}^{\infty} h_{n}(x) d \nu(x)=0 .
$$

Proof of Theorem 3. From Theorem 3 of [8],

$$
\left|a_{n}\right| \leq \frac{1}{2 \pi} \int_{0}^{2 \pi}\left|\frac{\left|\phi_{n}(z)\right|^{2}}{\left|\phi_{n+1}(z)\right|^{2}}-1\right| d \theta, \quad z=e^{i \theta} .
$$


The Cauchy inequality yields

$$
\left[\int_{0}^{2 \pi}\left|\frac{\left|\phi_{n}(z)\right|^{2}}{\left|\phi_{n+1}(z)\right|^{2}}-1\right| d \theta\right]^{2} \leq \int_{0}^{2 \pi}\left(\frac{\left|\phi_{n}(z)\right|}{\left|\phi_{n+1}(z)\right|}+1\right)^{2} d \theta \int_{0}^{2 \pi}\left(\frac{\left|\phi_{n}(z)\right|}{\left|\phi_{n+1}(z)\right|}-1\right)^{2} d \theta .
$$

Due to the well-known identity 3

$$
\int_{0}^{2 \pi} \frac{\left|\phi_{n}(z)\right|^{2}}{\left|\phi_{n+1}(z)\right|^{2}} d \theta=\int_{0}^{2 \pi}\left|\phi_{n}(z)\right|^{2} d \sigma(\theta)=2 \pi, z=e^{i \theta},
$$

we have an estimate

$$
\left|a_{n}\right|^{2} \leq 8\left[1-\frac{1}{2 \pi} \int_{0}^{2 \pi} \frac{\left|\phi_{n}(z)\right|}{\left|\phi_{n+1}(z)\right|} d \theta\right]
$$

We show that

$$
\left(\frac{|\mathbb{K}|}{2 \pi}\right)^{3 / 2} \leq \liminf _{n \rightarrow \infty} \frac{1}{2 \pi} \int_{0}^{2 \pi} \frac{\left|\phi_{n}(z)\right|}{\left|\phi_{n+1}(z)\right|} d \theta
$$

Then, the statement of the theorem will be immediate due to (91). Estimate (10), in turn, can be proved by the argument used in Theorem 3 of [5]. One needs to start with a fixed non-negative, continuous, $2 \pi$ periodic function $f(x)$. Then, Hölder's inequality yields

$$
\begin{aligned}
& {\left[\frac{1}{2 \pi} \int_{0}^{2 \pi}\left(\sigma^{\prime}(\theta) f(\theta)\right)^{1 / 4} d \theta\right]^{4}} \\
& \leq\left[\frac{1}{2 \pi} \int_{0}^{2 \pi} \frac{\left|\phi_{n}(z)\right|}{\left|\phi_{n+1}(z)\right|} d \theta\right]^{2}\left[\frac{1}{2 \pi} \int_{0}^{2 \pi}\left|\phi_{n+1}(z)\right|^{2} \sigma^{\prime}(\theta) d \theta\right]\left[\frac{1}{2 \pi} \int_{0}^{2 \pi} f(\theta)\left|\phi_{n}(z)\right|^{-2} d \theta\right] .
\end{aligned}
$$

The second factor is not greater than 1 ; measure $\left|\phi_{n}(\theta)\right|^{-2} d \theta$ converges weakly to $d \sigma$ [3]. Therefore,

$$
\begin{aligned}
& {\left[\frac{1}{2 \pi} \int_{0}^{2 \pi}\left(\sigma^{\prime}(\theta) f(\theta)\right)^{1 / 4} d \theta\right]^{4}} \\
& \quad \leq\left[\liminf _{n \rightarrow \infty} \frac{1}{2 \pi} \int_{0}^{2 \pi} \frac{\left|\phi_{n}(z)\right|}{\left|\phi_{n+1}(z)\right|} d \theta\right]^{2}\left[\frac{1}{2 \pi} \int_{0}^{2 \pi} f(\theta) d \sigma\right] .
\end{aligned}
$$

Assume that $\sigma$ has a singular component $\sigma_{s}$. Then, by Lemma 2, we can choose the sequence of $2 \pi$ periodic functions $\left\{h_{k}\right\}$ with the properties $0<h_{k} \leq 1, h_{k}(\theta) \rightarrow 1$ a.e.,

$$
\int_{0}^{2 \pi} h_{k}(\theta) d \sigma_{s}(\theta) \rightarrow 0
$$

as $k \rightarrow \infty$. If $\sigma$ is purely a.c., let $h_{k}(\theta) \equiv 1$ for all $k$. Fix any $\varepsilon>0$, then we can find the sequence of continuous, non-negative, $2 \pi$ periodic functions $g_{l}(\theta)$ such 
that $g_{l} \leq \varepsilon^{-1}, g_{l} \rightarrow\left(\sigma^{\prime}+\varepsilon\right)^{-1}$ a.e. For fixed $\varepsilon>0$, choose $f$ in (11) equal to $h_{k} g_{l}$. Taking $k \rightarrow \infty$, then $l \rightarrow \infty$, and, finally, $\varepsilon \rightarrow 0$, we get estimate (10).

Remark. That is not clear even for small values of $(2 \pi-|\mathbb{K}|)$ whether the estimate (8) is sharp or not.

Consider a bounded operator in $\ell^{2}\left(\mathbb{Z}^{+}\right)$given by a Jacobi matrix

$$
J=\left[\begin{array}{ccccc}
v_{0} & b_{0} & 0 & 0 & \ldots \\
b_{0} & v_{1} & b_{1} & 0 & \cdots \\
0 & b_{1} & v_{2} & b_{2} & \ldots \\
0 & 0 & b_{2} & v_{3} & \cdots \\
\cdots & \cdots & \cdots & \cdots & \cdots
\end{array}\right] .
$$

Theorem 4. Assume that $\sigma_{\text {ess }}(J)=[-1,1]$, and for the corresponding spectral measure $\mu(x)$, we have $\mu^{\prime}(x)>0$ for a.e. $x \in(-1,1)$. Then, $v_{k} \rightarrow 0$ and $b_{k} \rightarrow 1 / 2$ as $k \rightarrow \infty$.

Proof. Consider the sequence of matrices

$$
J_{n}=\left[\begin{array}{ccccc}
v_{n} & b_{n} & 0 & 0 & \cdots \\
b_{n} & v_{n+1} & b_{n+1} & 0 & \cdots \\
0 & b_{n+1} & v_{n+2} & b_{n+2} & \ldots \\
0 & 0 & b_{n+2} & v_{n+3} & \cdots \\
\cdots & \ldots & \cdots & \cdots & \cdots
\end{array}\right] .
$$

These are matrices of the operators generated by the quadratic forms $(J x, x)$, restricted to $x \in\left\{\left(x, e_{j}\right)=0, j=0,1, \ldots, n-1\right\}$, where $e_{j}=(0, \ldots, 0,1,0, \ldots)$ is the standard basis in $\ell^{2}\left(\mathbb{Z}^{+}\right)$. Matrix $J$ can be represented as the rank-two perturbation of the following orthogonal sum $J_{n} \oplus Q_{n}$, where $Q_{n}$ is the finite Jacobi matrix

$$
Q_{n}=\left[\begin{array}{ccccc}
v_{0} & b_{0} & 0 & \ldots & \ldots \\
b_{0} & v_{1} & b_{1} & \ldots & \ldots \\
0 & b_{1} & v_{2} & \ldots & \ldots \\
\ldots & \ldots & \ldots & \ldots & \ldots \\
0 & 0 & \ldots & b_{n-2} & v_{n-1}
\end{array}\right]
$$

From Weyl's theorem, we infer that $\sigma_{\text {ess }}\left(J_{n}\right)=[-1,1]$. The support of the a.c. component of the spectral measure is invariant under finite-rank perturbations. So, it is equal to $[-1,1]$ for any $J_{n}$. Due to Lemma 1 , we have $\inf \sigma\left(J_{n}\right) \rightarrow-1$, $\sup \sigma\left(J_{n}\right) \rightarrow 1$ as $n \rightarrow \infty$. That means $\left\|J_{n}\right\| \rightarrow 1$. Consider the matrix (operator) $\hat{J}_{n}=\left\|J_{n}\right\|^{-1} J_{n}$. Now, the spectral measure $d \psi^{(n)}$ of this operator is supported on the interval $[-1,1]$. Therefore, we can consider the corresponding system of polynomials orthogonal on the unit circle with measure $d \sigma^{(n)}$, given by (44), and the sequence of reflection parameters $\left\{a_{k}^{(n)}\right\}$. The complement in $[0,2 \pi]$ to the support of the a.c. part of the measure $d \sigma^{(n)}$ is the union of three intervals: $\left(0, \kappa_{1}^{(n)}\right)$, $\left(\pi-\kappa_{2}^{(n)}, \pi+\kappa_{2}^{(n)}\right)$ and $\left(2 \pi-\kappa_{1}^{(n)}, 2 \pi\right)$, where $\kappa_{1(2)}^{(n)} \rightarrow 0$ as $n \rightarrow \infty$. Therefore, Theorem 3 yields $\lim \sup _{k \rightarrow \infty}\left|a_{k}^{(n)}\right| \rightarrow 0$ as $n \rightarrow \infty$. Bearing in mind formulas (6), (77), and identity $\lim _{n \rightarrow \infty}\left\|J_{n}\right\|=1$, we have

$$
\limsup _{k \rightarrow \infty}\left|v_{k}\right|=0 \quad \text { and } \quad \limsup _{k \rightarrow \infty}\left|b_{k}-1 / 2\right|=0 .
$$


The idea of a truncation process was suggested by Yuditskii. Meanwhile, this approach is standard and was used before (see, e.g., [9]). The important analogs of Theorem 4 can be obtained for "continuous orthogonal systems" such as Krein systems, Dirac operators, one-dimensional Schrödinger operators. This is done in a separate publication [2].

\section{REFERENCES}

[1] N. I. Akhiezer, The Classical Moment Problem and Some Related Questions in Analysis, Hafner Publishing Company, New York, 1965. MR 32:1518

[2] S. A. Denisov, On the continuous analog of Rakhmanov's theorem for orthogonal polynomials, J. Funct. Anal. 198 (2003), 465-480.

[3] Y. L. Geronimus, Orthogonal Polynomials: Estimates, asymptotic formulas, and series of polynomials orthogonal on the unit circle and on an interval, Consultants Bureau, New York, 1961. MR 24:A3469

[4] Ya. L. Geronimus, Polynomials Orthogonal on a Circle and their Applications, Amer. Math. Soc. Transl. (1), Vol. 3, Providence, Rhode Island, 1954, 1-78. MR 15:869i

[5] A. Máté, P. Nevai, and V. Totik, Asymptotics for the ratio of leading coefficients of orthogonal polynomials on the unit circle, Constr. Approx. 1 (1985), 63-69. MR 85j:42045

[6] P. Nevai, Research problems in orthogonal polynomials, Approximation Theory 6, Vol. 2 (C. K. Chui et al., eds.), pp. 449-489, Academic Press, Boston, 1989. MR 91m:42023

[7] P. Nevai, Orthogonal polynomials, Memoirs Amer. Math. Soc., Vol. 18, No. 213, Amer. Math. Soc., Providence, RI, 1979. MR 80k:42025

[8] P. Nevai, Weakly convergent sequences of functions and orthogonal polynomials, J. Approx. Theory 65 (1991) No. 3, 322-340. MR 92f:42031

[9] E. M. Nikishin, The discrete Sturm-Liouville operator and some problems of function theory, Trudy Sem. Petrovsk. 10 (1984), 3-77 (in Russian), J. Soviet Math. 35 (1986), 2679-2744. MR 86h:39007

[10] E. A. Rakhmanov, On the asymptotics of the ratio of orthogonal polynomials. II, Math. USSR Sb. 46 (1983), 105-117.

[11] F. Riesz and B. Sz.-Nagy, Functional Analysis, Frederick Ungar Publishing, New York, 1955. MR 17:175i

[12] G. Szegö, Orthogonal Polynomials, Amer. Math. Soc. Colloquium Publications, Vol. 23, Providence, RI, 1975. MR 51:8724

Department of Mathematics, California institute of Technology, 253-37, Pasadena, CALifornia 91125

E-mail address: denissov@caltech.edu 